\title{
The Effect of Sound Level on Perception of Reproduced Soundscapes
}

Authors: Anugrah Sabdono Sudarsono; Yiu W Lam; William J Davies

Email: a.s.sudarsono@edu.salford.ac.uk, y.w.lam@salford.ac.uk, W.Davies@salford.ac.uk.

The aim of this work was to investigate the perception of soundscape reproduced by an ambisonic reproduction system on a horizontal plane, how the experience of space affected the perception of soundscape reproduction, and how the sound level adjustment on soundscape reproduction affected the perception of soundscape compared with actual conditions. There were three experiments conducted: a soundwalk in situ in Manchester (United Kingdom) city centre, listening tests in Salford (United Kingdom), and listening tests in Bandung (Indonesia). The listening tests used material recorded from four locations on the soundwalk route in Manchester. The Salford listening tests were performed at the in-situ measured sound level, and the participants were asked to adjust the sound level to the level that represents actual locations. The listening test in Bandung was conducted to understand the effect of participants who never come to the actual location to the perception of soundscape and the sound level adjustment. The listening tests in Bandung were conducted at the in situ sound level, at $9.5 \mathrm{~dB}$ below the in situ sound level (based on the preference sound level from the experiment in Salford), and the participants were also requested to adjust the sound level to the level that represents the actual space (to examine the consistency with the experiment in Salford). In each case, soundscape perception was measured on 19 semantic differential scales. Analysis of the semantic differential results showed that the ambisonic reproduction produced a similar subjective experience to the in situ soundwalk when the reproduction sound level was $9.5 \mathrm{~dB}$ lower than the actual sound level in situ. Reproduction at the actual sound level in situ produced a different dimensional space. The study shows that the sound level adjustment of soundscape reproduction in laboratory experiment produces more ecologically valid results compared to the reproduction at the actual sound level in situ.

\section{Introduction}

Audio reproduction systems are often used to recreate an outdoor soundscape in the laboratory for subjective testing. Several methods have analysed the validity of various reproductions to reproduce soundscape. For instance, Guastavino and Katz tried to compare 
stereo, ambisonic system on the horizontal plane, and ambisonic system with height (threedimensional ambisonic system) to reproduce soundscape in an anechoic condition (Guastavino \& Katz, 2004). Five scales were applied for the experiment: Readability, Presence, Distance, Localization, Coloration, and Stability. Furthermore, this experiment confirms that ambisonic system on the horizontal plane could reproduce proper spatial aspect of soundscape on the sweet spot, and suitable for outdoor soundscape reproduction.

The different method also conducted to validate the ambisonic reproduction system in reproducing outdoor soundscape in laboratory. Semantic categorization from verbal response has been adapted to compare soundscape reproduction between the stereo system, ambisonic system, and actual condition (Guastavino, Katz, Polack, \& Levitin, 2005). Three categories regarding the response were used here: Source, Object-Centered, and Subject-Centered. The experiment shows that ambisonic reproduction in anechoic condition with the speakers conceals from the view enabled the participants to feel that they were in real locations. Although the ambisonic reproduction system appears to offer better reproduction, many other experiments of soundscape reproduction were conducted using binaural system (Axelsson, Nilsson, \& Berglund, 2010)(Cain, Jennings, \& Poxon, 2013)(Hall, Irwin, Edmondson-Jones, Phillips, \& Poxon, 2013).

Davies et al. conducted one of the studies regarding soundscape reproduction in the laboratory that used ambisonic system and the system confirmed the similar result of Semantic Differential Analysis with the in situ condition (Davies, Bruce, \& Murphy, 2014). In their study, three-dimensional ambisonic reproduction system was implemented to reproduce soundscape in the semi-anechoic chamber (Davies et al., 2014). Four perceptual dimensions were established from this experiment: Relaxation/Calmness, Dynamic/Vibrancy, Communication, and Spatiality. The perceptual similarity of this reproduction was confirmed by comparing with the result of field experiment in Sheffield (Kang, 2007). The dimensions gathered in the laboratory showed similar dimensions compared to the in situ experiment: Relaxation, Communication, Spatiality, and Dynamic. In another word, the three-dimensional ambisonic playback systems in the semi-anechoic chamber could give a similar impression with the actual condition although the information regarding the sound level of reproduction was not well defined.

Interestingly, in the previous work (Davies et al., 2014), it was found that the participants tended to lower the sound level of event sound objects (not the overall sound level) in the 
soundscape simulator by $-12.3 \mathrm{~dB}$ in average from the recording level. The soundscape simulator allowed the participants to compose a soundscape by adjusting the sound level of each sound object in a soundscape. Although the sound level adjustment might indicates that the participant might prefer lower sound level reproduction, this study has not analysed the overall reproduction sound level of simulated soundscape and the effect of the sound level adjustment on the perception of soundscape reproduced in the laboratory.

In this work, the validity of soundscape reproduction using the two-dimensional ambisonic systems was analysed. Two-dimensional ambisonic reproduction obviously offers much simpler set up than the three-dimensional ambisonic reproduction while at the same time could still reproduce better outdoor soundscape (Guastavino \& Katz, 2004). Also, the study about the overall sound level adjustment of soundscape reproduction, and how the sound level adjustment on soundscape reproduction affected the perception of soundscape compared with actual conditions will be analysed further.

\section{Method}

\subsection{Soundscape Recording}

The soundscape was recorded using a soundfield microphone in the Manchester city centre area. The Soundfield ST-250 microphone was used with Roland R-44 digital recorder that recorded all four outputs (W, X, Y, and Z signal) from the microphone simultaneously. The windshield was applied to the microphone to reduce wind noise. The recordings were taken for ten minutes at each location in a stationary condition.

The Manchester city centre soundscapes were recorded at several outdoor locations: National Football Museum, Exchange Square, New Cathedral Street, St Ann Square, Market Street, and Piccadilly Garden. All of the recordings were made in February 2014 during the lunchtime. Four recordings were selected for the experiment: Market Street as a representation of busy shopping spot, St Ann Square due to the tranquillity, Piccadilly Garden as the icon of Manchester city centre, and food market at Piccadilly Garden due to the different function of space. A snapshot of the locations is shown in Figure 1. The snapshot is also indicating the typical sound in each place: People walking and talking on Market Street, the water fountain at St Ann Square, a combination of people and urban traffic at Piccadilly Garden, and the sound of food stalls at food market at Piccadilly Garden. The audio samples, two minutes long, were selected from each of recordings. The samples were chosen to 
represent each soundscape based on completeness of the sound components, and the occurrence of wind noise in the recording.
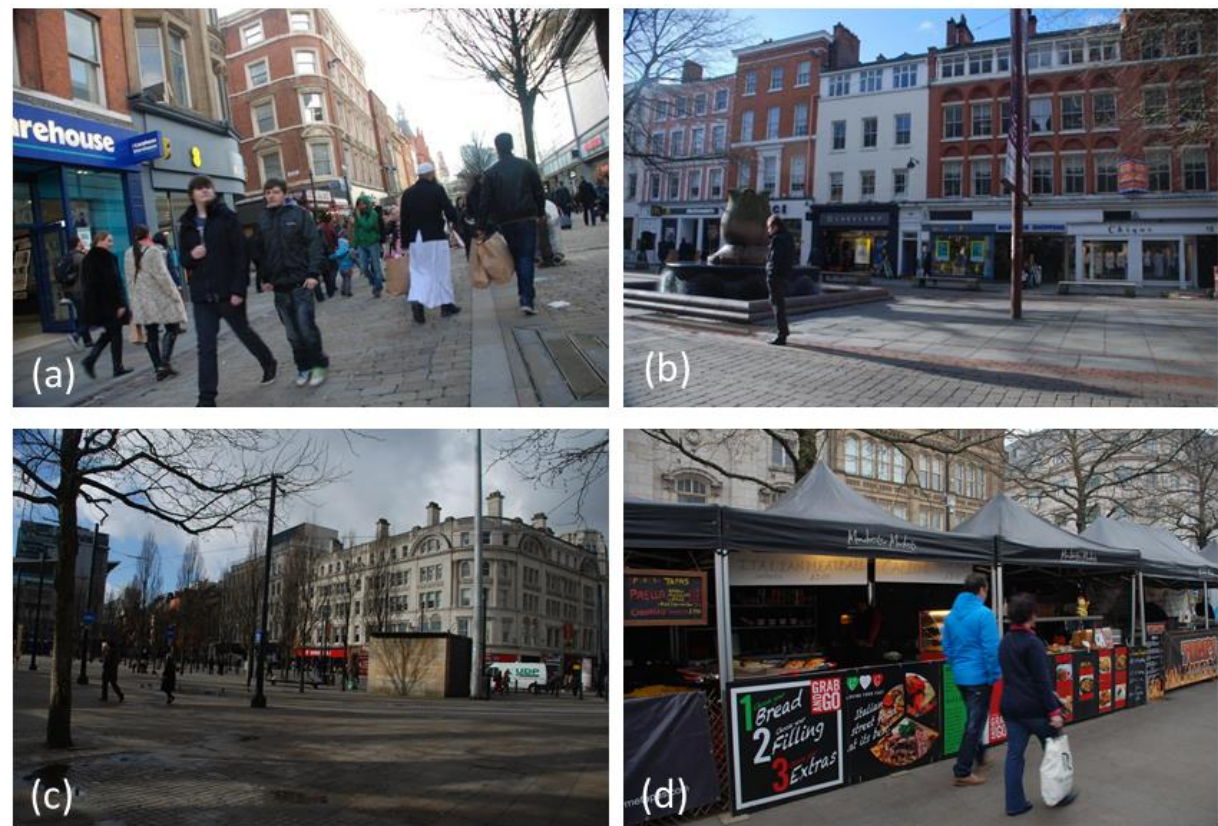

Figure 1 Snapshot of the locations; (a) Market Street; (b) St Ann Square; (c) Piccadilly

\section{Garden; and (d) Food Market at Piccadilly Garden}

Four soundscape recordings were used in these experiments, and the Sound Pressure Level (SPL) measurement of the locations are shown in Table 5. The SPL data were calculated from the $\mathrm{W}$ channel from the soundfield microphone that had been calibrated. The data of $\mathrm{L}_{10}$ (the SPL surpassed 10\% of recording time ), L50 (the SPL surpassed 50\% of recording time), and $\mathrm{L}_{90}$ (the SPL surpassed $90 \%$ of recording time) was also calculated from each of recordings. The noisiest location was Market Street ( $73 \mathrm{dBA})$, and the quietest location was St Ann Square (62 dBA). Both the recordings made in Piccadilly Garden have a similar condition with the noise level at $70 \mathrm{dBA}$.

Table 1 Noise Measurement of Recordings

\begin{tabular}{|l|c|c|c|c|}
\hline & Market Street & St Ann Square & Piccadilly Garden & Food Market at Piccadilly Garden \\
\hline L10 (dBA) & 74 & 65 & 73 & 72 \\
\hline L50 (dBA) & 73 & 62 & 70 & 69 \\
\hline L90 (dBA) & 71 & 60 & 68 & 67 \\
\hline Leq (dBA) & 73 & 62 & 70 & 70 \\
\hline
\end{tabular}




\subsection{Experiment}

There were three experiments to verify the validity of soundscape reproduction in a room. The first experiment was conducted in a Listening Room at the University of Salford, United Kingdom. The second experiment was performed in a recording room at Institut Teknologi Bandung, Indonesia. The third experiment was carried out at Manchester City Centre, United Kingdom. The experiment in Bandung was conducted to understand how the participants who never come to the actual locations would adjust the sound level of soundscape reproduction, and how would they rate the soundscape of places that never been visited before. Analysis, using semantic differential analysis with Principal Component Analysis (PCA), also performed to understand the effect of two different participant experiences: first, the participants in Salford who are familiar with the actual soundscape location; and the participants in Bandung, who have never visited the actual location.

All the experiments were carried out using semantic questionnaire with the scale developed at the University of Salford (Davies et al., 2014). These scales are closely based on those of Kang (2007). All the scales were represented as eleven point scale with the description shown in Table 2 as anchor points.

Table 2 Semantic Differential Scales

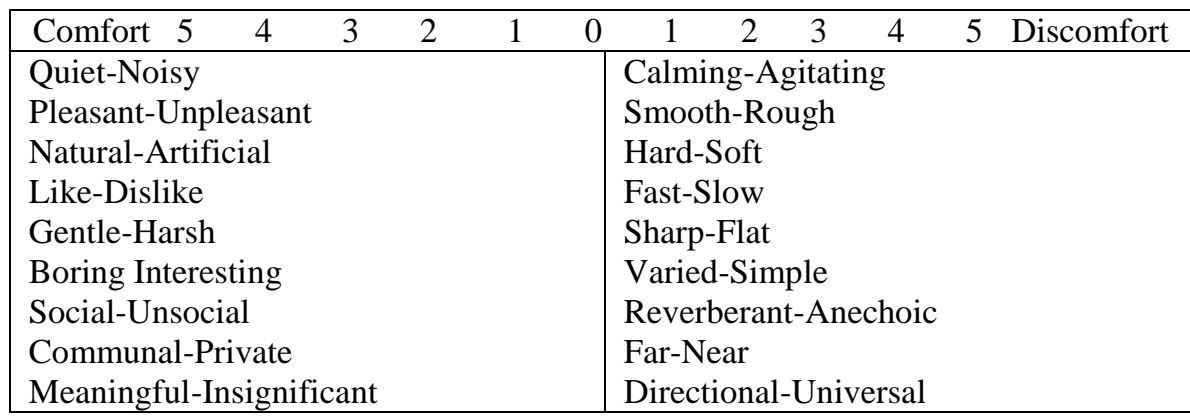

\subsection{Laboratory Experiment in Salford}

\subsubsection{Experiment Set Up}

The listening test was done with two systems: audio playback system, and audio control system. Audio playback systems consisted of eight Genelec 1029A speakers connected with RMA ADI-8DS and M-Audio Profire Lightbridge Audio Interface. A Behringer BCR 2000 Digital Audio Workstation (DAW) controller was used to control the sound level of the audio playback with Reaper DAW software. In this listening test, the soundscape recording was reproduced using Wig Ware Ambisonic Decoder developed by Bruce Wiggins (Wiggins, 2010). Near field compensation was applied in this system. The listening test was conducted 
in a listening room at the University of Salford that meets the requirement of BS 684013 / IEC 268-13.

\subsubsection{Experiment Method and Participants}

The experiment was carried out individually in four sessions, and a practice session before the experiment began. The experiment was last for thirty minutes. All the participants were asked to listen to the soundscape and imagine themselves in the actual place. The soundscape recordings were reproduced randomly in each session without telling the locations where the recordings were made. Eighteen participants participated in this listening test. Most of them were Master or Ph.D. students at the University of Salford with various backgrounds (acoustic, audio engineering, engineering, and social science) and ethnicity (Asian, British, European, and African). There were 14 male and four female participants with the age range between 24-40 years old. All of the participants joined the experiment voluntarily.

The semantic differential scale with 11 points was used to rate the soundscape based on participants impression of the soundscape. In each session, the participants were asked to fill the semantic questionnaire while listening to a soundscape, and the participants were requested to adjust the sound level of reproduction to the sound level that they think represent the actual sound level at the actual locations after they finish filling the questionnaire.

\subsection{Laboratory Experiment in Bandung}

\subsubsection{Experiment Set Up}

This listening test was conducted in a recording room at Institut Teknologi Bandung, Indonesia. Eight KRK Rockit 5 speakers were used with the 2D ambisonic playback system. A laptop with Reaper DAW software was connected to M-Audio Fast Track Ultra 8R audio interface. Wig Ware Ambisonic Decoder developed by Bruce Wiggins (Wiggins, 2010) with near field compensation was used to decode B-format recording of Manchester City Centre. A DAW controller, Korg Nanokontrol 2, was used to control the level of the reproduction.

\subsubsection{Experiment Method and Participants}

Two experiments were conducting in Bandung. The first experiment was carried on with the same method with the experiment in Salford (listening to the soundscapes at the actual sound level, rate the soundscapes, and adjust the sound level of reproduction). In the first experiment, fifteen participants (eight males and seven females) joined the experiment voluntarily. All of the participants were bachelor and master students in Engineering Physics 
with an age range between 17-34 years old. The experiment was done individually and last for thirty minutes.

The second experiment was conducted by reproducing the soundscape at $-9.5 \mathrm{~dB}$ below the actual sound level. The value was based on the result of the experiment in Salford. Sixteen different participants (nine males, and seven females) from the previous experiment joined the experiment voluntarily. All of the participants were bachelor and master students in Engineering Physics with an age range between 18-29 years old. The experiment was done individually and last for thirty minutes.

The questionnaire used in this experiment was the same semantic scales utilised in the experiment in Salford. All the participants were explained about the scale before the experiment to familiarised the scale.

\subsection{In Situ Experiment}

\subsubsection{Experiment Method and Participants}

In situ experiments were conducted with soundwalk, and fill the semantic scale at the location where the recording of laboratory test was made. The soundwalk commonly carried out in a group (Adams et al., 2008)(Liu, Kang, Behm, \& Luo, 2014) and the participants were asked to listen to the soundscape silently. The soundwalk was done in four locations in the city centre: Piccadilly Garden, Food Market in Piccadilly Garden, Market Street, and St Ann Square. The locations are shown in Figure 2.

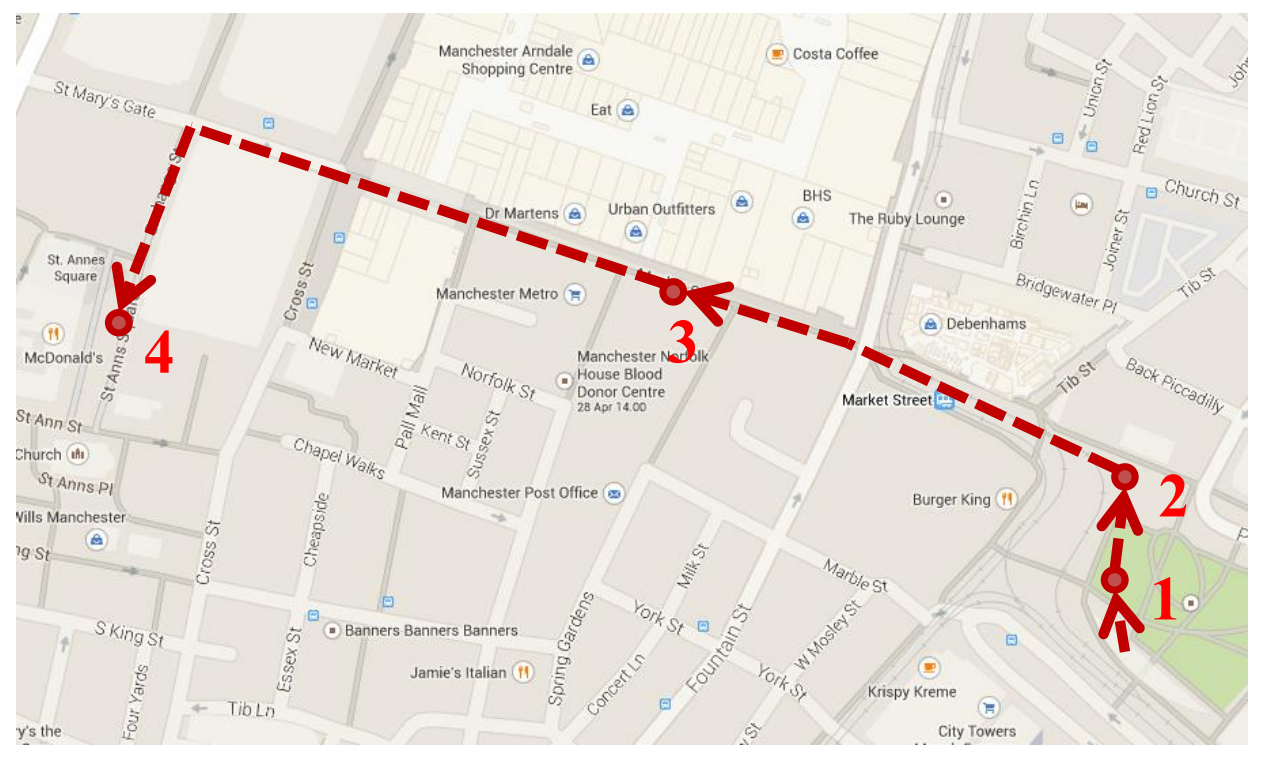

Figure 2 Locations and Soundwalk Route 
The experiment was conducted in four sessions with 23 participants: 22 January 2015 (ten participants, five males and five females), 24 January 2015 (three participants, all males), 29 January 2015 (one participant, male), and 31 January 2015 (nine participants, five males and four females). The age of the participants was between 23 to 50 years old and came from different ethnicity (Asian, British, and European). The snapshot of the experiment is shown in Figure 3.

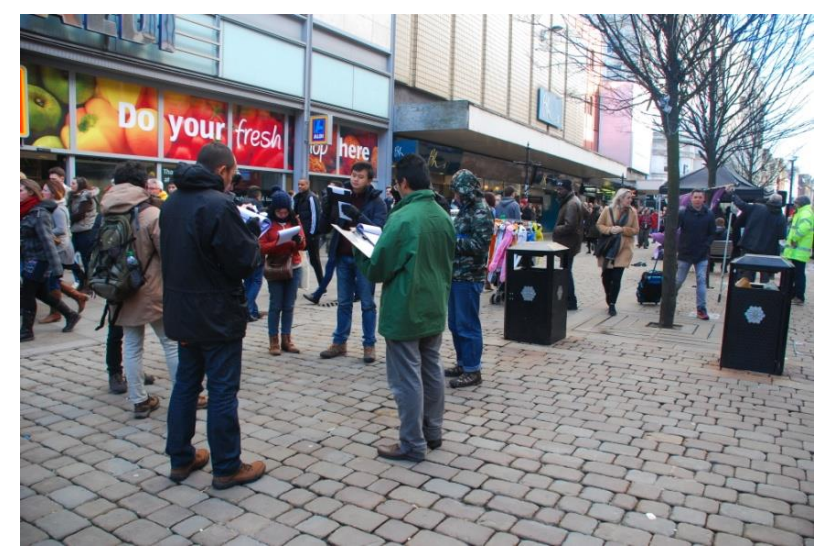

Figure 3 Soundwalk at Manchester City Centre

\section{Results and discussion}

\subsection{Assessment of Soundscape Reproduction in Laboratory}

Soundscape reproduction with first order ambisonic with eight speakers in a Listening Room was analysed using semantic differential analysis to understand the impression of the soundscape.

PCA was used to analyse the semantic data. The significant components from PCA were determined based on the eigenvalue of the components (eigenvalue $>1$ ) and further analysis was done using reliability test.

\subsubsection{In-Situ Experiment}

Factor analysis for the in-situ dataset was done by combining the result of semantic scales from the four locations. The PCA from in situ experiment, as shown in Table 3, showed six components explain $72 \%$ of variance in the scale:

- Component 1 (24\%) called Calmness/Relaxation. The scale of ComfortDiscomfort, Quiet-Noisy, Pleasant-Unpleasant, Like-Dislike, Gentle-Harsh, and Smooth-Rough load highly into this component. 
- Component 2 (14\%) called Dynamic/Vibrancy. The scale of Hard-Soft, Fast-Slow, Sharp-Flats, and Varied-Simple load highly into this component.

- Component $3(11 \%)$ called Communication. The scale of Social-Unsocial and Communal-Private load highly into this component.

- Component 4 (9\%) called Naturality and Meaningful. The scale of MeaningfulInsignificant, and Natural-Artificial load highly into this component.

- Component 5 (7\%) called Spatialy. The scale of Reverberant-Anechoic loads highly into this component.

- Component 6 (7\%) called Directivity.The scale of Directional-Universal loads highly into this component.

Table 3 PCA of In Situ Experiment

PCA In Situ (N= 92, Kaiser-Mayer-Olkin index =0.647, Bartlett's test of sphericity sig. 0.000)

\begin{tabular}{|c|c|c|c|c|c|c|}
\hline & \multicolumn{7}{|c|}{ Component } \\
\cline { 2 - 7 } & $24 \%$ & $14 \%$ & $11 \%$ & $9 \%$ & $7 \%$ & $7 \%$ \\
\hline Comfort- Discomfort & .826 & -.105 & -.036 & -.203 & -.074 & .052 \\
\hline Quiet-Noisy & .640 & .016 & -.375 & -.132 & .080 & -.345 \\
\hline Pleasant-Unpleasant & .891 & -.066 & .057 & .046 & -.035 & .195 \\
\hline Natural-Artificial & .519 & .138 & -.088 & .538 & -.228 & -.183 \\
\hline Like-Dislike & .861 & -.041 & .144 & .138 & -.119 & .218 \\
\hline Gentle-Harsh & .713 & -.491 & -.128 & .031 & .073 & -.068 \\
\hline Boring-Interesting & -.475 & -.292 & .029 & -.311 & .075 & -.412 \\
\hline Social-Unsocial & .096 & .332 & .790 & .049 & .039 & -.017 \\
\hline Communal-Private & -.135 & -.025 & .872 & .165 & -.013 & -.179 \\
\hline Meaningful-Insignificant & .457 & .190 & .315 & .573 & -.078 & .170 \\
\hline Calming-Agitating & .458 & -.180 & -.309 & .109 & .464 & -.069 \\
\hline Smooth-Rough & .515 & -.622 & -.054 & .003 & .294 & -.093 \\
\hline Hard-Soft & -.354 & .642 & .233 & -.123 & .115 & .189 \\
\hline Fast-Slow & -.073 & .502 & .231 & .266 & .408 & .262 \\
\hline Sharp-Flats & .029 & .851 & -.055 & .085 & .203 & -.084 \\
\hline Varied-Simple & .112 & .621 & .414 & .067 & -.207 & -.169 \\
\hline Reverberant-Anechoic & -.138 & .119 & .019 & -.021 & .817 & .066 \\
\hline Far-Near & .279 & .065 & -.160 & -.829 & -.168 & -.013 \\
\hline Directional-Universal & .138 & -.024 & -.184 & -.029 & .096 & .808 \\
\hline
\end{tabular}

Reliability test (using Cronbach's Alpha) indicates that only three dimensions are reliable to measure the soundscapes: Calmness/Relaxation (Cronbach's Alpha $=0.872)$, Dynamic $($ Cronbach's Alpha $=0.818)$, and Communication $($ Cronbach's Alpha $=0.706)$. The test demonstrates that although there are six dimensions gathered from the semantic differential analysis, only three dimensions are reliable. Further investigations are conducted with the focus on those three dimensions. 
The in situ experiment give similar results to the field studies at urban locations in Sheffield (Kang, 2007) that confirm four main dimensions: Relaxation (26\%), Communication (12\%), Spatiality (8\%), and Dynamic (7\%). The entire dimensions of Kang's experiment also appear in our experiment. The dimension of Calmness/Relaxation in our experiment seems to explain similar variance with Kang's experiment (24\% in our experiment, and $26 \%$ in Kang's experiment). The variance value is also similar with the dimension of Communication (11\% in our experiment, and 12\% in Kang's experiment). The other dimensions of Kang's (Dynamic and Spatiality) are shown in our experiment with a higher percentage of variance.

The in situ experiment also showed a similar dimension to the field study in France (Raimbault, Lavandier, \& Bérengier, 2003). The study suggests that there are three dimensions of soundscape: Assessment and Strength (67\%), Sound Dynamic (15\%), and Spatial Dimension and Clarity (8\%). The dimension of Calmness/Relaxation in our experiment is alike with the dimension of assessment and strength. The dimension of sound dynamic also appears in our experiment and explained similar variance (14\% in our experiment and $15 \%$ in the experiment in France).

\subsubsection{Laboratory Test at Actual Sound Level Reproduction}

Analysis of soundscape reproduction at actual sound level was conducted using the data from the experiment in Salford and Bandung. Factor analysis of a laboratory experiment in Salford dataset was done by combining the result of semantic scales from four experiment sessions. The PCA from laboratory experiment in Salford, as shown in Table 4, showed five components explain $69 \%$ of variance in the scale:

- Component 1 (25\%) called Calmness/Relaxation. The scale of ComfortDiscomfort, Quiet-Noisy, Pleasant-Unpleasant, Like-Dislike, Gentle-Harsh, CalmingAgitating, and Smooth-Rough load highly into this component.

- Component 2 (14\%) called Communication and Dynamic. The scale of SocialUnsocial, Hard-Soft, Fast-Slow, Sharp-Flats, and Varied-Simple load highly into this component.

- Component 3 (12\%) called Spatiality. The scale of, Reverberant-Anechoic, and FarNear load highly into this component.

- Component 4 (9\%) called Naturality and Meaningful. The scale of MeaningfulInsignificant, and Natural-Artificial load highly into this component. 
- Component 5 (6\%) called Directivity. The scale of Directional-Universal loads highly into this component.

Table 4 PCA of Laboratory Test in Salford at Actual Level Reproduction

PCA Laboratory Salford Actual Level (N=54, Kaiser-Mayer-Olkin index 0.754, Bartlett's test of sphericity sig. 0.000 )

\begin{tabular}{|c|c|c|c|c|c|}
\hline & \multicolumn{5}{c|}{ Component } \\
\cline { 2 - 6 } & $25 \%$ & $17 \%$ & $12 \%$ & $9 \%$ & $6 \%$ \\
\hline Comfort- Discomfort & .828 & .053 & .092 & .147 & .137 \\
\hline Quiet-Noisy & .688 & -.305 & .068 & .136 & .033 \\
\hline Pleasant-Unpleasant & .754 & .057 & -.178 & .389 & .194 \\
\hline Natural-Artificial & .215 & -.182 & -.156 & .735 & -.027 \\
\hline Like-Dislike & .715 & .146 & -.202 & .490 & .154 \\
\hline Gentle-Harsh & .841 & -.083 & .186 & .265 & -.112 \\
\hline Boring-Interesting & -.386 & -.490 & .423 & -.090 & -.177 \\
\hline Social-Unsocial & .017 & .521 & -.469 & -.019 & -.157 \\
\hline Communal-Private & -.011 & .404 & -.606 & -.039 & -.295 \\
\hline Meaningful-Insignificant & .172 & .405 & -.085 & .728 & -.031 \\
\hline Calming-Agitating & .778 & -.221 & .073 & -.237 & .006 \\
\hline Smooth-Rough & .760 & -.374 & -.040 & .023 & -.071 \\
\hline Hard-Soft & -.582 & .562 & .068 & .041 & .294 \\
\hline Fast-Slow & -.209 & .787 & -.146 & .014 & .046 \\
\hline Sharp-Flats & -.272 & .689 & .147 & .194 & -.102 \\
\hline Varied-Simple & -.118 & .712 & -.236 & -.067 & -.276 \\
\hline Reverberant-Anechoic & .101 & .051 & .739 & -.232 & .052 \\
\hline Far-Near & .026 & -.079 & .827 & -.063 & -.042 \\
\hline Directional-Universal & .070 & -.146 & .106 & -.031 & .898 \\
\hline
\end{tabular}

The PCA from the laboratory experiment in Salford show different results with the experiment conducted outdoors at Manchester City Centre. The four dimensions of space that proposed by Kang (2007) exist in the laboratory experiment, but with the dimension of Communication and Dynamic unite into one dimension. The components related to Calmness/Relaxation, Naturality and Meaningful, and Directivity show the same outcome with the in situ experiment. Those components were formed from the identical semantic scales, and also showed the matching amount of variance explanation of the PCA.

The difference appears in the second, and the third components. The second component in the laboratory experiment (Communication and Dynamic) shows the combination of the second component (Dynamic) and the third component (Communication) from in situ experiment. This combination shows that the participants respond the soundscape reproduction differently from the real condition. The experiment using three-dimensional ambisonic systems (Davies et al., 2014) shows that the dimension of Dynamic and Communication were separated, 
although the soundscape was reproduced in the laboratory. Unfortunately, the information about the reproduction level of soundscape reproduction is not clearly informed in the study.

The experiment in Bandung was conducted to understand how the perception of people who never come to a certain place perceives the soundscape reproduction of that space. The analysis was done using factor analysis. The result of PCA is shown in Table $\mathbf{5}$ and the analysis indicates four main dimensions that explain $74 \%$ of all variations:

- Component 1 (32\%) called Calmness/Relaxation. the scale of Comfort- Discomfort, Pleasant-Unpleasant, Like-Dislike, Gentle-Harsh, Meaningful-Significant, CalmingAgitating, and Smooth-Rough load highly into this component.

- Component 2 (26\%) called Communication and Dynamic. The scale of SocialUnsocial, Communal-Private, Fast-Slow, Sharp-Flats, and Varied-Simple load highly into this component.

- Component 3 (9\%) called Spatially. The scale of Natural-Artifical and Far-Near load highly into this component.

- Component 4 (7\%) called Directivity. The scale of Directional-Universal loads highly into this component.

The first, second, and the fourth components seem consistent with the result of the experiment in Salford. The combination of Communication and Dynamic in this experiment indicates the difference of participants' perception with the perception of actual location. 
Table 5 PCA of Laboratory Test in Bandung at Actual Level Reproduction

PCA Laboratory Bandung Actual Level (N=60, Kaiser-Mayer-Olkin index 0.810, Bartlett's test of sphericity sig. 0.000)

\begin{tabular}{|c|c|c|c|c|}
\hline & \multicolumn{4}{|c|}{ Component } \\
\hline & $32 \%$ & $26 \%$ & $9 \%$ & $7 \%$ \\
\hline Comfort- Discomfort & .917 & -.067 & .022 & .068 \\
\hline Quiet-Noisy & .450 & -.636 & .263 & .073 \\
\hline Pleasant-Unpleasant & .850 & -.124 & .039 & .151 \\
\hline Natural-Artificial & -.045 & .082 & .775 & .166 \\
\hline Like-Dislike & .932 & -.013 & .045 & -.003 \\
\hline Gentle-Harsh & .761 & -.294 & .188 & .013 \\
\hline Boring-Interesting & -.722 & -.345 & .141 & .052 \\
\hline Social-Unsocial & .040 & .850 & .133 & .115 \\
\hline Communal-Private & .000 & .864 & .123 & .052 \\
\hline Meaningfull-Insignificant & .660 & .072 & -.289 & -.151 \\
\hline Calming-Agitating & .771 & -.419 & -.034 & -.089 \\
\hline Smooth-Rough & .788 & -.296 & -.038 & -.033 \\
\hline Hard-Soft & -.654 & .499 & .047 & .117 \\
\hline Fast-Slow & -.475 & .624 & -.104 & .147 \\
\hline Sharp-Flat & -.205 & .677 & -.316 & -.044 \\
\hline Varied-Simple & -.083 & .848 & -.158 & .024 \\
\hline Reverberant-Anechoic & .030 & -.050 & -.148 & -.862 \\
\hline Far-Near & -.072 & -.300 & .669 & -.330 \\
\hline Directional-Universal & -.012 & .076 & -.427 & .561 \\
\hline
\end{tabular}

Reliability test from two laboratory experiments on normal level reproduction in Salford and Bandung indicates that only two dimensions that reliable to measure the soundscapes:

Calmness/Relaxation (Cronbach's Alpha $=0.906$ and 0.930), and the combination of Communication and Dynamic (Cronbach's Alpha $=0.791$ and 0.747). The test demonstrates that the laboratory experiment in Bandung gives a similar result with the experiment in Salford (although the experiment in Bandung showed more variation). Furthermore, it indicates that the experience of space is not affected the judgement a soundscape.

The result of the laboratory experiments without sound level adjustment indicating similar results with the other studies in the laboratory. The study conducted by Axelsson et al.(2010) using headphone shows three significant soundscape dimensions: pleasantness (50\%), eventfulness (18\%), and familiarity (6\%) (Axelsson et al., 2010). Another study by Cain et.al (2013) using headphone shows two significant soundscape dimensions: calmness (60\%), and vibrancy (20\%) (Cain et al., 2013). Two significant soundscape dimensions (Pleasantness, calmness and intrusiveness (24\%); and vibrancy and informational content (24\%)) shown in the study by Hall et al. (2013) using headphone (Hall et al., 2013). Another study conducted using 4 speakers system indicates three soundscapes dimensions: Emotional Assessment and Strength Factor (42\%), Activity (14\%), and Clarity (10\%) (Guillén \& López Barrio, 2007). Two dimensions are consistent with all the studies, including from our study: the dimension 
associated with the impression of general assessment such as calmness and pleasantness; and the dimensions associated with the feeling of vibrancy, dynamic and activity.

Our experiment shows that soundscape reproduction using two-dimensional ambisonic playback systems at actual sound level cause the perception difference between laboratory condition and in situ condition (the dimensions of Communication and Dynamic combine into one scale in laboratory condition while the in situ experiment shows that the two dimensions become different dimensions). It might suggest that the 2D ambisonic system without sound level adjustment could not reproduce the similar feeling of the outdoor soundscape.

\subsection{The Effect of Sound level Adjustment on Perception of Soundscape}

\section{Reproduction}

The effect of sound level adjustment on perception was analysed in two steps: first, by determined the sound level adjustment of reproduced soundscape by participants in the laboratory (Experiment in Salford and Bandung); and second, by comparing the perception of soundscape reproduced in the laboratory (with and without sound level adjustment) with the in situ soundwalk.

\subsubsection{Sound level Adjustment}

The analysis of sound level adjustment was based on the experiment by Davies et al.(2014) using soundscape simulator (Davies et al., 2014). The participants of their study tend to lower the sound objects by $-12.3 \mathrm{~dB}$ from the recording sound level in the soundscape simulator. The sound level adjustment indicates that the overall soundscape simulated by the simulator should be reproduced at a lower sound level than the actual level.

The laboratory experiments were conducted (using four soundscape recording) in Salford, and Bandung. The participants were asked to adjust the sound level to the level that represents real condition. The experiment confirmed that if the participants have an opportunity to adjust the sound level of reproduction, they tend to lower the reproduction level than the actual level.

The sound level adjustment of every location in the laboratory was analysed by Analysis of Variance (ANOVA) with $95 \%$ significance level. The ANOVA shows that there is no significant difference between all the recordings used with the sound level adjustment. This result indicates that the amount of sound level adjustment is unrelated with the loudness of 
the reproduced soundscape. In brief, the participants adjusted the reproduced soundscape with the similar value of adjustment as shown in Figure 4 although the soundscapes had different loudness.

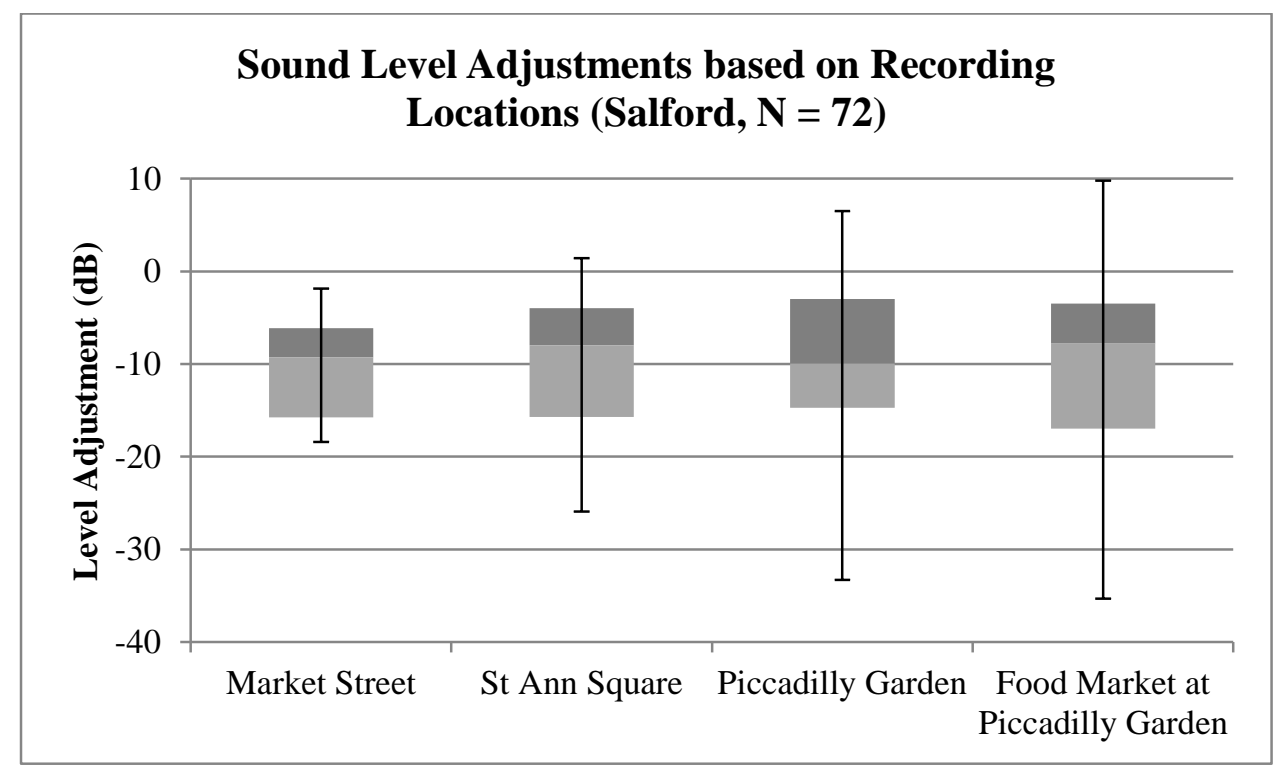

Figure 4 Sound level Adjustments of Soundscape Reproduction based on Recording Locations on the Experiment in Salford

Analysis of expert and non-expert was conducted to understand if there were any differences between expert and non-expert participants in adjusting the sound level. Expert participants were the participants who have experience in acoustic or audio engineering for more than five years and has participated in at least five listening test before this experiment. They were eight participants who were considered as experts based on our criteria. The analysis was done using Mann-Whitney test and show that the difference of group is not significant, but the expert group show lower variance than the non-expert as shown in Figure 5. 


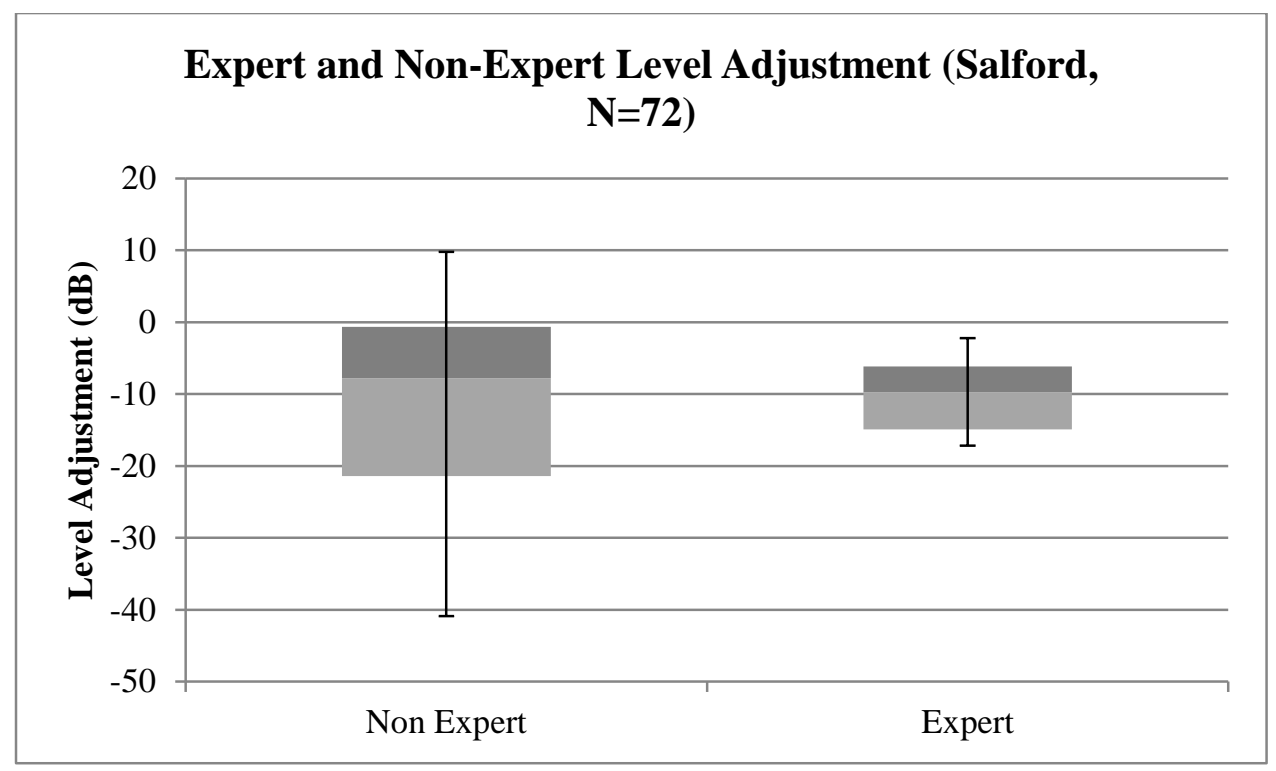

Figure 5 Expert and Non-Expert Level Adjustment of Soundscape Reproduction on the Experiment in Salford

Some non-expert participants had adjusted the sound level to the level that they felt comfortable, and they adjusted it very low (one participant adjusts the sound level to $-49 \mathrm{~dB}$ ), but overall the adjustment was consistent at the same level of adjustment. The overall sound level adjustment of soundscape reproduction in the laboratory is consistent with the level of $9.5 \mathrm{~dB}$ (based on median calculation) as shown in Figure 6.

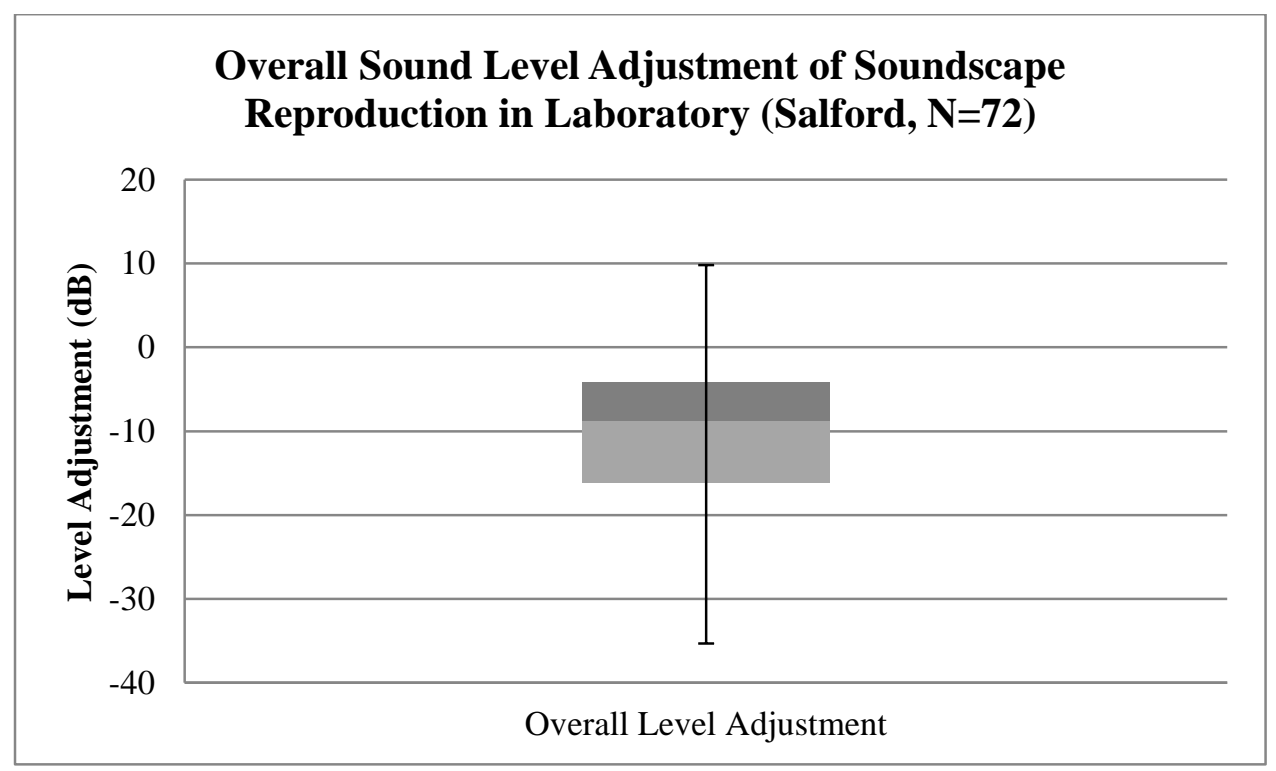

Figure 6 Overall Sound Level Adjustment of Soundscape Reproduction on the Experiment in Salford

\subsubsection{Level of Adjustment Difference between Experiment in Salford and Bandung}


The experiment in Bandung was conducted to verify the level of sound level adjustment by participants, and to understand how the impression of soundscape reproduction that was reproduced by $-9.5 \mathrm{~dB}$ below the actual sound level (based on the previous experiment in Salford).

Sound level adjustment of soundscape reproduction was conducted by asking the participants to adjust the sound level to the level that represents the actual condition. The result is shown in Figure 7. ANOVA test was used to analyse the effect of different recording accustomed in the sound level adjustment experiment and showed that there is no significant difference ( $>0.05)$ due to the variation of soundscape recording. The participants have a tendency to adjust the sound level about $-9.5 \mathrm{~dB}$ from the real level outdoor. This result is consistent with the previous study conducted in Salford.

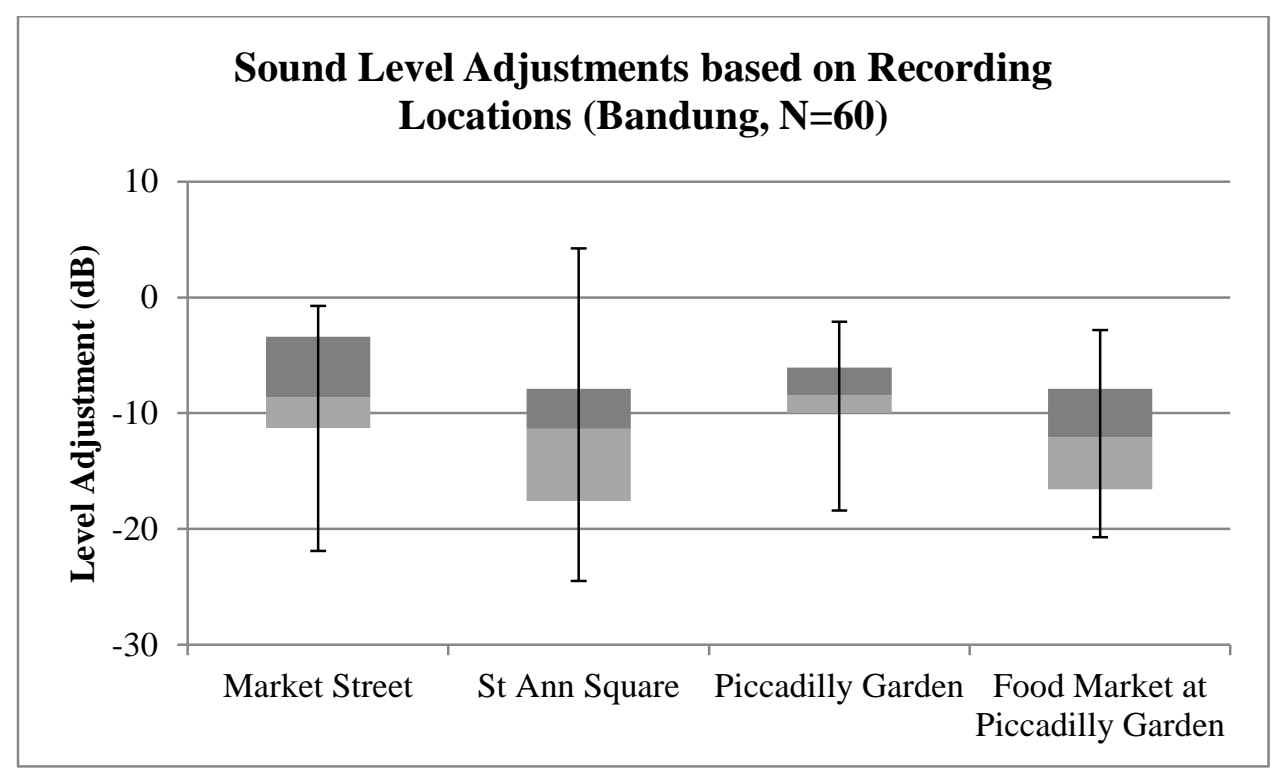

Figure 7 Sound Level Adjustments of Soundscape Reproduction based on Recording Locations on the Experiment in Bandung

The data from this experiment were also compared with the experiment conducted in Salford to analyse the effect of different participant's background, and room condition with the sound level adjustment. The experiment in Salford was carried out in a listening room with people who lived in Manchester. The experiment in Bandung was conducted in a normal recording room with Indonesian participants. The experiment showed that there is no significant different $(p>0.05)$ of sound level adjustment from the laboratory experiment in Salford and Bandung as illustrated in Figure 8. The adjustment was at $-9.5 \mathrm{~dB}$ on both the laboratory experiment in Salford and Bandung. 


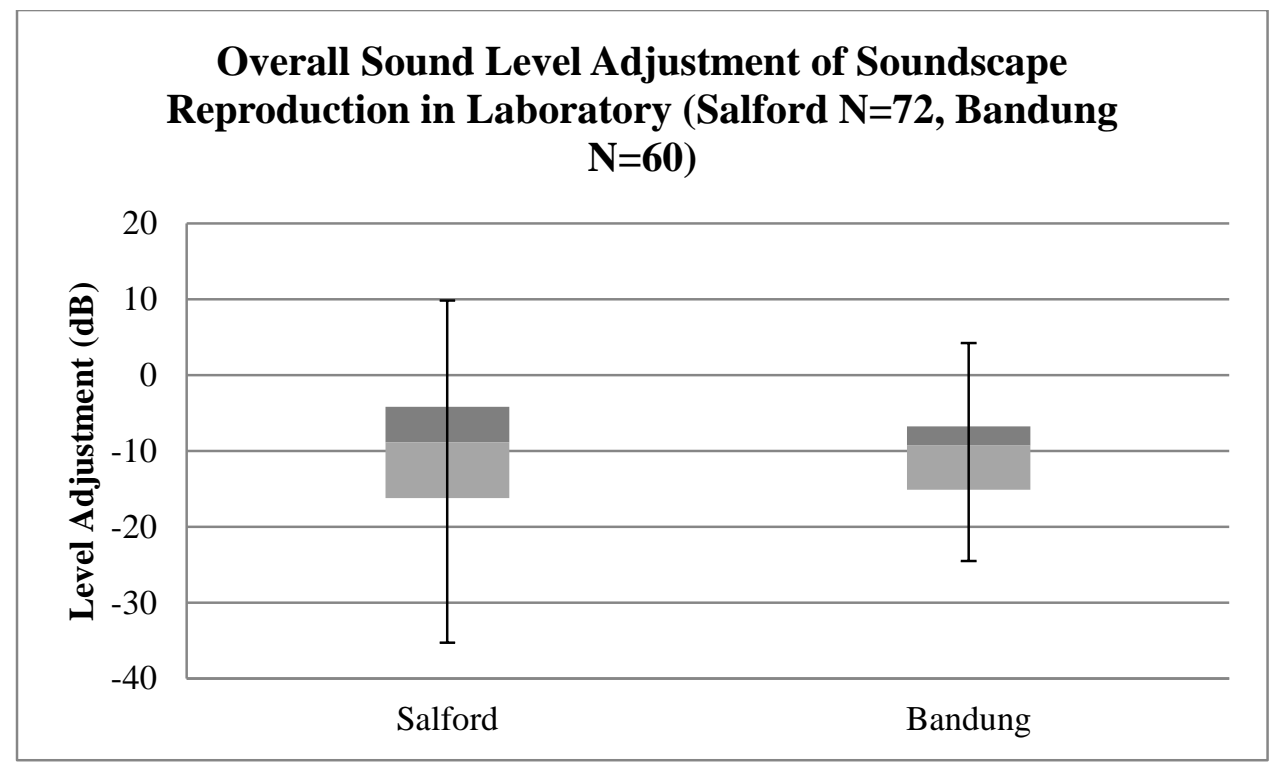

Figure 8 Overall Sound level Adjustment of Soundscape Reproduction on the Experiment in Salford and Bandung

\subsubsection{Semantic Differential Analysis between In Situ Experiment and Laboratory Experiment with Sound Level Adjustment}

Factor analysis of experimental data set was done by combining the result of semantic scales from four locations. The PCA from laboratory experiment with $-9.5 \mathrm{~dB}$ sound level adjustment, as shown in Table 6, showed five components explain $76 \%$ of variance in the scale:

- Component $1(32 \%)$ called Calmness/Relaxation. The scale of ComfortDiscomfort, Quiet-Noisy, Pleasant-Unpleasant, Like-Dislike, Gentle-Harsh, Meaningful-Insignificant, and Smooth-Rough load highly into this component.

- Component 2 (18\%) called Dynamic/Vibrancy. The scale of Fast-Slow, Sharp-Flats, and Varied-Simple load highly into this component.

- Component $3(12 \%)$ called Communication. The scale of Social-Unsocial, and Communal-Private load highly into this component.

- Component 4 (7\%) called Spatiality. The scale of Reverberant-Anechoic and FarNear load highly into this component.

- Component 5 (7\%) called Directivity. The scale of Directional-Universal loads highly into this component. 
Table 6 PCA of the Experiment in Bandung with -9.5 dB Sound Level Adjustment

PCA Laboratory Bandung with -9.5 dB Sound Level Adjustment (N= 64, Kaiser-Mayer-Olkin index 0.790, Bartlett's test of sphericity sig. 0.000$)$

\begin{tabular}{|c|c|c|c|c|c|}
\hline & \multicolumn{5}{|c|}{ Component } \\
\cline { 2 - 5 } & $32 \%$ & $18 \%$ & $12 \%$ & $7 \%$ & $7 \%$ \\
\hline Comfort- Discomfort & .873 & .143 & .006 & -.066 & -.017 \\
\hline Quiet-Noisy & .619 & -.387 & -.437 & .005 & -.045 \\
\hline Pleasant-Unpleasant & .907 & .078 & -.006 & -.087 & .090 \\
\hline Natural-Artificial & .138 & -.608 & .427 & -.284 & .271 \\
\hline Like-Dislike & .843 & .167 & .223 & -.087 & .052 \\
\hline Gentle-Harsh & .799 & -.252 & -.096 & .184 & .101 \\
\hline Boring-Interesting & -.357 & -.694 & -.330 & .071 & .155 \\
\hline Social-Unsocial & -.102 & .045 & .872 & -.065 & -.084 \\
\hline Communal-Private & -.198 & .356 & .719 & .168 & -.367 \\
\hline Meaningful-Insignificant & .546 & .473 & .307 & .018 & .190 \\
\hline Calming-Agitating & .819 & -.159 & -.194 & .003 & .166 \\
\hline Smooth-Rough & .794 & -.197 & -.094 & .160 & -.067 \\
\hline Hard-Soft & -.821 & .022 & .206 & -.206 & .162 \\
\hline Fast-Slow & -.577 & .583 & .136 & -.049 & .219 \\
\hline Sharp-Flats & -.093 & .794 & -.029 & -.038 & .117 \\
\hline Varied-Simple & -.098 & .775 & .367 & .078 & .183 \\
\hline Reverberant-Anechoic & .099 & .197 & -.027 & .835 & -.129 \\
\hline Far-Near & .060 & -.476 & .058 & .653 & .294 \\
\hline Directional-Universal & .041 & .161 & -.190 & -.001 & .873 \\
\hline
\end{tabular}

The soundscape reproduction with $-9.5 \mathrm{~dB}$ sound level adjustment shows the similar result with the field study conducted by Kang (2007), the laboratory experiment using threedimensional ambisonic system by Davies et al. (2014), and the in situ experiment. The first three dimensions are the same dimension from the in situ experiment. The dimensions of Dynamic and Communication, which combine into one dimension with the reproduction at the actual sound level, are separated into two dimensions as occur in the field experiment. Furthermore, based on reliability test using Cronbach's Alpha, the reliable dimensions that occur in field test also reliable in this experiment (Calmness/Relaxation $=0.918$, Dynamic $=$ 0.738, and Communication= 0.756). In conclusion, the soundscape reproduction with -9.5 sound level adjustments could give the similar impression with the in-situ experiment rather than using soundscape reproduction with actual sound level. The finding also suggests the reason for the sound level adjustment: the participants might feel that the reproduction at actual sound level is not ecologically realistic. 


\section{Conclusion}

The soundscape reproduction using two-dimensional ambisonic reproduction system with at actual sound level could not produce a similar impression of soundscape at actual conditions based on Semantic Differential Analysis. When people have the opportunity to adjust the sound level of soundscape reproduction in the laboratory, they tend to adjust the sound level to $-9.5 \mathrm{~dB}$ below the actual level. The adjustment was consistent, although the experiments were conducted with participants with different experiences of actual locations, and different type of room used for experiments (listening room in Salford, and recording room in Bandung). Furthermore, the soundscape reproduction using two-dimensional ambisonic system with $-9.5 \mathrm{~dB}$ sound level adjustment seems to be a better approach for soundscape reproduction in a room because it could give more similar perception with the perception of the actual soundscape.

\section{Acknowledgement}

This work is part of a study sponsored by the Indonesia Endowment Fund for Education, Ministry of Finance (LPDP), Indonesia. Many thanks to Joko Sarwono as the head of Building Physics and Acoustic Laboratory, Institut Teknologi Bandung for giving permission to use the laboratory facility.

\section{Reference}

Adams, M., Bruce, N., Davies, W., Cain, R., Jennings, P., Carlyle, A., ... Plack, C. (2008). SOUNDWALKING AS METHODOLOGY FOR UNDERSTANDING SOUNDSCAPES. In Institute of Acoustics Spring Conference 2008 (Vol. 30). Reading: the Institute of Acoustics. Retrieved from http://usir.salford.ac.uk/2461/

Axelsson, Ö., Nilsson, M. E., \& Berglund, B. (2010). A principal components model of soundscape perception. The Journal of the Acoustical Society of America, 128(5), 283646. http://doi.org/10.1121/1.3493436

Cain, R., Jennings, P., \& Poxon, J. (2013). The development and application of the emotional dimensions of a soundscape. Applied Acoustics, 74(2), 232-239. http://doi.org/10.1016/j.apacoust.2011.11.006

Davies, W. J., Bruce, N. S., \& Murphy, J. E. (2014). Soundscape Reproduction and Synthesis. Acta Acustica United with Acustica, 100(2), 285-292. http://doi.org/10.3813/AAA.918708

Guastavino, C., \& Katz, B. F. G. (2004). Perceptual evaluation of multi-dimensional spatial 
audio reproduction. The Journal of the Acoustical Society of America, 116(2), 1105. http://doi.org/10.1121/1.1763973

Guastavino, C., Katz, B. F. G., Polack, J., \& Levitin, D. J. (2005). Ecological Validity of Soundscape Reproduction. Acta Acoustica United with Acoustica, 91(September 2004), 333-341. Retrieved from http://www.ingentaconnect.com/content/dav/aaua/2005/00000091/00000002/art00015

Guillén, J. D., \& López Barrio, I. (2007). The soundscape experience. In 19th INTERNATIONAL CONGRESS ON ACOUSTICS. Sociedad Española de Acústica. Retrieved from http://hdl.handle.net/10261/5298

Hall, D. a., Irwin, A., Edmondson-Jones, M., Phillips, S., \& Poxon, J. E. W. (2013). An exploratory evaluation of perceptual, psychoacoustic and acoustical properties of urban soundscapes. Applied Acoustics, 74(2), 248-254. http://doi.org/10.1016/j.apacoust.2011.03.006

Kang, J. (2007). Urban Sound Environment (1st ed.). Oxon: Taylor and Francis.

Liu, J., Kang, J., Behm, H., \& Luo, T. (2014). Effects of landscape on soundscape perception: Soundwalks in city parks. Landscape and Urban Planning, 123, 30-40. http://doi.org/10.1016/j.landurbplan.2013.12.003

Raimbault, M., Lavandier, C., \& Bérengier, M. (2003). Ambient sound assessment of urban environments: field studies in two French cities. Applied Acoustics, 64(12), 1241-1256. http://doi.org/10.1016/S0003-682X(03)00061-6

Wiggins, B. (2010). The Blog of Bruce: WigWare. Retrieved from http://www.brucewiggins.co.uk/?page_id=78 